\title{
ANALYSIS OF INCIDENCE AND RISK FACTORS OF RETINOPATHY OF PREMATURITY AMONG VERY-LOW-BIRTH-WEIGHT INFANTS IN NORTH TAIWAN

\author{
C.-Y. Yang
} \\ Division of Neonatology, Depts. of Pediatrics, Chang Gung Children's Hospital, Chang Gung University College of Medicine, Kwei-Shan Township, Taiwan R.O.C.
}

Background: Although significant advances have been made in perinatal care, retinopathy of prematurity (ROP) remains a serious complication in prematurely born individuals. There have been limited studies on ROP in Taiwan, and most of those existing reports are out-dated.

Methods: This retrospective study included 252 very-low-birth-weight (VLBW) infants admitted to the neonatal intensive care unit (NICU) of Chang Gung Children's Hospital over a 2-year period between July 2005 and June 2007. All infants were examined for ROP according to the guidelines published by the American Academy of Pediatrics (AAP). The relationship between clinical risk factors and the development of ROP was analyzed.

Results: Of the 252 VLBW infants, 216 met the screening criteria. Of the 216, 99 (45.8\%) had ROP. The simultaneous presence of a low GA, low birth weight (BW), lower Apgar scores, hypotension, patent ductus arteriosus (PDA), septicemia, intraventricular hemorrhage (IVH), ventilator dependence, and use of postnatal steroids was associated with severe ROP. Using multiple logistic regression analyses for ROP, only maternal preeclampsia [odds ratio (OR), 2.52; confidence interval (CI), 1.32-4.7], duration of mechanical ventilation (OR, 1.06; CI, 1.04-1.08), and LBW (OR, 2.62; CI, 1.370-3.375) predicted the development of threshold ROP.

Conclusion: The incidence of ROP among VLBW infants was 45.8\%; 19.0\% had severe ROP. Infants of lower GAs and/or with low BW, whose mother had preeclampsia, or who had a long duration of mechanical ventilation are at risk for the development of threshold ROP. 